\title{
A Novel System to Study Dengue Virus Replication Organelle Formation Independent from Viral RNA Replication $^{\dagger}$
}

\author{
Berati Cerikan 1, Sarah Goellner ${ }^{1}$, Christopher John Neufeldt ${ }^{1}$, Uta Haselmann ${ }^{1}$, Mirko Cortese ${ }^{1}$ \\ and Ralf Bartenschlager 1,2,* \\ 1 Department of Infectious Diseases, Molecular Virology, University of Heidelberg, 69120 Heidelberg, \\ Germany; Berati.Cerikan@med.uni-heidelberg.de (B.C.); Sarah.Goellner@med.uni-heidelberg.de (S.G.); \\ Christopher.Neufeldt@med.uni-heidelberg.de (C.J.N.); Uta.Haselmann@med.uni-heidelberg.de (U.H.); \\ Mirko.Cortese@med.uni-heidelberg.de (M.C.) \\ 2 German Center for Infection Research (DZIF), Heidelberg Partner Site, 69120 Heidelberg, Germany \\ * Correspondence: Ralf.Bartenschlager@med.uni-heidelberg.de \\ † Presented at Viruses 2020-Novel Concepts in Virology, 5-7 February 2020.
}

Published: 7 August 2020

\begin{abstract}
Positive-strand RNA viruses, such as dengue virus (DENV), induce the extensive rearrangement of intracellular membranes that serve as a scaffold for the assembly of the viral replication machinery. In the case of DENV, the main endomembrane ultrastructure produced in infected cells consists of invaginations of the endoplasmic reticulum, designated vesicle packets (VPs), which are the assumed sites of viral RNA replication. VPs are observed as arrays of vesicles surrounded by an outer membrane, the formation of which is induced by the viral nonstructural proteins, presumably in conjunction with specific host factors. However, little is known about the mechanisms governing VP formation, which is mainly due to the lack of a replication-independent system supporting the biogenesis of these membranous structures. Here we describe an expression-based, viral RNA replication-independent, DENV polyprotein system, designated as pIRO (plasmid-induced replication organelle), which is sufficient to induce VP formation. We show that VPs induced by pIRO expression are morphologically indistinguishable from those found in infected cells, suggesting that DENV replication organelle formation does not require RNA replication. We conclude that the pIRO system is a novel and valuable tool that can be used to dissect the mechanisms underlying DENV replication organelle formation.
\end{abstract}

Keywords: flavivirus; vesicle packet; replication organelle; organelle biogenesis; membrane invagination; membranous organelle; viral replicase; replication complex

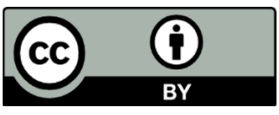

(C) 2020 by the authors. Licensee MDPI, Basel, Switzerland. This article is an open access article distributed under the terms and conditions of the Creative Commons Attribution (CC BY) license (http://creativecommons.org/licenses/by/4.0/). 